\title{
Subcellular resolution 3D light field imaging with genetically encoded voltage indicators
}

\author{
Peter Quicke $^{\mathrm{a}, \mathrm{b}}$, Carmel L. Howe ${ }^{\mathrm{a}, \mathrm{b}}$, Pingfan Song ${ }^{\mathrm{c}}$, Herman Verinaz Jadan ${ }^{\mathrm{c}}$, Chenchen \\ Song $^{\mathrm{d}}$, Thomas Knöpfel ${ }^{\mathrm{d}, \mathrm{b}}$, Mark Neil ${ }^{\mathrm{e}, \mathrm{b}}$, Pier Luigi Dragotti ${ }^{\mathrm{c}}$, Simon R. Schultz ${ }^{\mathrm{a}, \mathrm{b}^{*}}$, Amanda \\ J. Foust tab** $^{\text {, }}$ \\ ${ }^{a}$ Department of Bioengineering, Imperial College London, London, UK \\ ${ }^{\mathrm{b}}$ Centre for Neurotechnology, Imperial College London, London, UK \\ ${ }^{c}$ Department of Electrical and Electronic Engineering, Imperial College London, London, UK \\ ${ }^{\mathrm{d}}$ Department of Brain Sciences, Imperial College London, London, UK \\ ${ }^{\mathrm{e}}$ Department of Physics, Imperial College London, London, UK
}

\begin{abstract}
Light field microscopy (LFM) enables high signal-to-noise ratio (SNR), light efficient volume imaging at fast frame rates, and has been successfully applied to single-cell resolution functional neuronal calcium imaging. Voltage imaging with genetically encoded voltage indicators (GEVIs) stands to particularly benefit from light field microscopy's volumetric imaging capability due to high required sampling rates, and limited probe brightness and functional sensitivity. Previous LFM studies have imaged GEVIs to track population-level interactions only in invertebrate preparations and without single cell resolution. Here we demonstrate sub-cellular resolution GEVI light field imaging in acute mouse brain slices resolving dendritic voltage signals localized in three dimensions. We characterize the effects of different light field reconstruction techniques on the SNR and signal localization and compare the SNR to fluorescence transients imaged in wide field. Our results demonstrate the potential of light field voltage imaging for studying dendritic integration and action potential propagation and backpropagation in 3 spatial dimensions.
\end{abstract}

Keywords: light field microscopy, genetically encoded voltage indicator, voltage imaging.

*Simon R. Schultz, s.schultz@ic.ac.uk **Amanda J. Foust, a.foust@ic.ac.uk

\section{$1 \quad 1$ Introduction}

2 Cellular resolution voltage imaging enables direct observation of neuronal computation. Indeed,

3 membrane potential imaging experiments have spatiotemporally resolved both active and passive

4 action and synaptic potential generation throughout dendritic and axonal arbors. ${ }^{1-14}$ Resolution

5 of these small voltage signals at high speeds requires high photon fluxes, making wide field sin-

6 gle photon (1P) imaging by far the most common voltage imaging modality. Imaging neuronal

7 processes with this technique requires the imaged membranes to lie approximately flat in the mi-

8 croscope's focal plane. As these experiments are typically performed in slices, the requirement

9 for flat, healthy, and superficial cells represents a significant barrier to entry for experimenters. 
10 Even in the best-prepared slices, anatomy dictates that only a few cells will be oriented parallel to

11 the surface, reducing experimental throughput, and only certain cell types feature morphology that

12 can be well-sampled by a single plane. Multiple approaches to improving wide field imaging's

13 3D performance have been developed. Anselmi et al. (2011) ${ }^{15}$ applied remote focusing to axially

14 shift and tilt the wide field focal plane as required by the sample, enabling calcium imaging along

15 tilted dendrites. This adaptation, however, costs half of the fluorescence emission and is limited to

16 a single tilted plane at a time. Point spread function (PSF) engineering via cubic phase masks ${ }^{16}$ or

17 spherical aberration ${ }^{17}$ also enables parallelised volumetric sample imaging when combined with

18 light sheet excitation, however to our knowledge these approaches have not successfully been im-

19 plemented to image membrane voltage.

20 Lack of optical sectioning with wide field 1P imaging further complicates matters. Light from

21 out-of-focus structures pollutes in-focus signals, confounding allocation of signals to axially sep-

22 arated processes. This issue is difficult to resolve with traditional optically sectioning confocal

23 or two-photon microscopy approaches as they are point scanning. Sequential sampling of each

24 pixel greatly reduces imaging bandwidth, and the fast frame rates required for voltage imaging

25 necessitates short dwell times and therefore few collected photons. This restricts Poisson-noise

26 limited SNR to low levels, making point scanning voltage imaging applicable to a limited number

27 of experimental paradigms. ${ }^{12,18-20}$

28 Fluorescence excitation parallelization into multiple spots, ${ }^{21-27}$ blobs, ${ }^{28,29}$ lines, ${ }^{30-32}$ sheets, ${ }^{33-40}$

29 or specified patterns $s^{41-45}$ increases the photon budget, enabling functional volumetric imaging or

30 single-plane imaging at increased speeds. A small number of these have been applied to imaging

31 voltage in 2 dimensions, ${ }^{26,31,42,45}$ however they are not able to image neuronal processes in $3 \mathrm{D}$.

32 Many of these techniques also trade-off reduced robustness to scattering compared to single-point 
33 scanning modalities for the increased excitation from parallelization.

34 Parallelized 3D two-photon imaging with elongated Bessel ${ }^{46,47}$ or stereoscopic tilted ${ }^{48}$ beams

35 excites narrow columns of fluorescence and relies on temporal and spatial sparsity of labelling and

36 activity to demix time courses from different $\mathrm{z}$ planes. This increases the volume rate but each

37 columnar pixel is still addressed sequentially, limiting bandwidth. These techniques have been

38 used to image calcium fluorescence transients but not yet voltage. images, extending wide field imaging whilst maintaining its unparalleled fluorescence excitation and collection efficiency. This is achieved by inserting a microlens array (MLA) at the native image plane of the microscope and placing the image sensor at its back focal plane (Fig. 1a).

43 This disperses the angular components of the collected image (Fig. 1b), which can be used to 44 infer objects' axial positions. Each LFM image consists of circular subimages (Fig. 1c), with 45 each subimage resembling a pixel in an undersampled image of the scene. Within each circular 46 subimage, each pixel location encodes a different angular sampling through the object intersecting

47 with the subimage's location, a columnar tomographic projection through the sample. ${ }^{50}$ Light field 48 images are typically parameterized by the 4D function $\mathcal{L}(u, v, x, y)$, where each lenslet subimage 49 is $\mathcal{L}(u, v, \cdot, \cdot)$ and the same specific pixel under each subimage is $\mathcal{L}(\cdot, \cdot, x, y)$. The "native $\mathrm{LFM}$ 50 resolution' with which the object is laterally sampled is given by the microlens pitch divided by 51 the objective magnification, much worse than the corresponding wide field resolution. In exchange

52 the microlenses provide angular information that can be used to render views of the object from 53 different perspectives, focus on different planes, and reconstruct 3D volumes all from a single 2D 54 frame. This technique converts a key disadvantage of wide field 1P fluorescence excitation, lack 55 of optical sectioning, into an advantage, as out-of-focus light renders 3D information about the 
sample.

Two prominent algorithms for reconstructing source volumes from LFM images have been described, synthetic refocusing ${ }^{49}$ and $3 \mathrm{D}$ deconvolution. ${ }^{50}$ Synthetic refocusing relies on a ray optics model of LFM image formation to reconstruct images at the native LFM resolution equivalent to those of a wide field microscope focused at any axial plane in the sample. Focal stacks can be generated similar to standard microscope z-stacks by combining images reconstructed at multiple axial depths. Each pixel in the refocused image is a weighted sum of light field image pixels, meaning refocusing is fast. The reconstructed images, however, suffer from the same blur due to lack of optical sectioning as a standard wide field microscope.

An alternative approach is based on reconstruction of the source volume using a forward model of light-field image formation (the LFM PSF) based on wave optics. ${ }^{50}$ Iterative deconvolution approaches such as Richardson-Lucy (RL) $)^{51,52}$ or the Image Space Reconstruction Algorithm $(\text { ISRA })^{53}$ find the maximum likelihood source volume given the measured image and LFM PSF in the presence of Poissonian (RL) or Gaussian (ISRA) noise. This approach is able to reconstruct source volumes at a lateral resolution greater than the native LFM resolution (the MLA pitch in the sample) by leveraging the fine sampling of the LFM tomographic projections. ${ }^{50}$ This increased resolution reconstruction fails where the tomographic sampling is degenerate, most notably around the native focal plane of the microscope, although newer designs have circumvented this limitation. ${ }^{54-57}$ The source volume is also reconstructed with less axial blur than in the refocused case, increasing axial signal discriminability.

Electrical length constants in neurons are on the scale of tens to hundreds of microns, making 7 increased lateral pixel size less disadvantageous for voltage imaging. Over-resolving electrical 8 fluctuations by imaging at or below the diffraction limit is typically unnecessary and can even hurt 
79 SNR by increasing the relative impact of non-Poisson noise such as read noise. Spatial resolu-

80 tion is therefore often sacrificed in voltage imaging experiments to increase speed or SNR. Many

81 such experiments use low read noise, high sensitivity CCD sensors featuring low pixel counts,

82 with pixels often measuring several microns across in the sample plane. Even with higher pixel

83 count detectors, the relatively low sensitivity of many voltage probes means multiple pixels are

84 often binned to increase SNR to acceptable levels. LFM's decreased native lateral sampling rate

85 therefore suits voltage imaging well, and deconvolution of LFM voltage imaging time series can

86 be implemented without oversampling to reduce computational cost.

87 LFM has successfully imaged calcium over large volumes in C. elegans and zebrafish, ${ }^{58,59}$ and

88 in both head-fixed and behaving mice. ${ }^{60-62}$ Voltage dynamics have also been imaged successfully

89 without single-cell resolution in Drosophila ${ }^{63}$ and larval zebrafish ${ }^{64}$ as part of whole brain imaging

90 setups alongside calcium imaging. LFM has not, to our knowledge, been applied to studying sub-

91 cellular or single-cell resolution voltage dynamics in any sample, despite its apparent suitability. In

92 this study we apply LFM to sub-cellular GEVI imaging in acute mouse brain slices. We combine

93 this technique with a recently reported transgenic strategy driving sparse expression in a random

94 subset of layer $2 / 3$ cortical pyramidal neurons which enables the resolution of single-cell level

95 voltage signals in neuronal somata and dendrites. ${ }^{65,66}$

96 We demonstrate that LFM is able to simultaneously image axially separated dendrites, enabling

97 single-shot capture and localisation of GEVI fluorescence transients in the 3D dendritic arbour.

98 We compare and evaluate deconvolution and synthetic refocusing for different GEVI imaging ap-

99 plications, whilst using a coarse deconvolution approach with no lateral oversampling to reduce

100 computational cost. We also apply a recently developed LFM PSF calculation ${ }^{67}$ for high NA ob-

101 jectives. We show that LFM enables 3D localization of dendritic and somatic GEVI fluorescence 

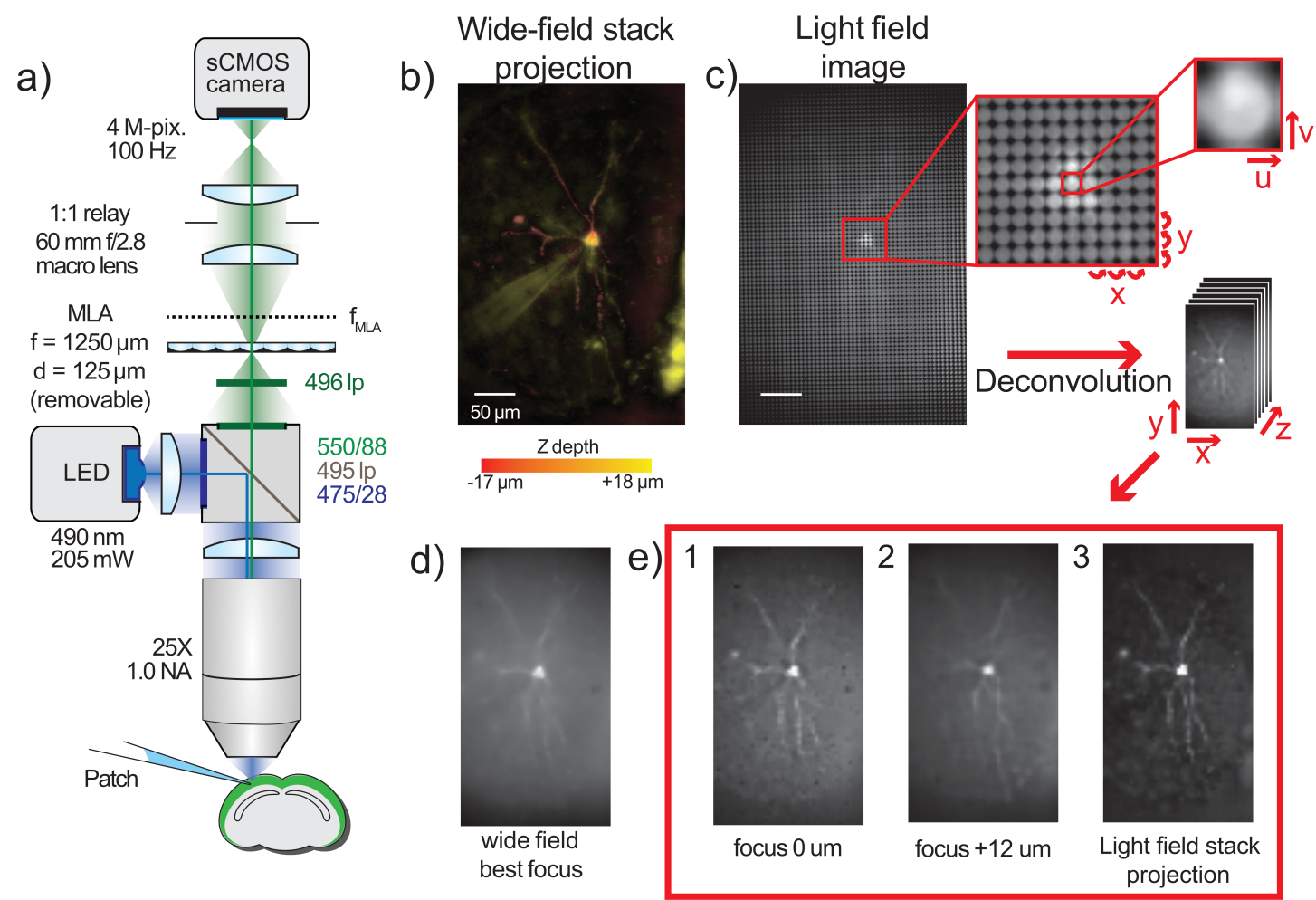

Fig 1 Light field microscopy enables simultaneous focusing on axially separated dendrites. a) LFM Diagram b) A pseudocolour z-projection of a wide field image stack through a GEVI labelled cell. Depth is color coded from red (superficial) to yellow (deep). Individual dendrites follow tortuous paths in all 3 dimensions, making simultaneous focussing on them all impossible in wide field microscopy. c) A light field image of the same cell showing the structure of light field images. Each spot in the light field image is a spatial sampling (coordinates $\mathrm{x}, \mathrm{y}$ ) of the angular distribution of rays (coordinates $\mathrm{u}, \mathrm{v}$ ) at that point. This angular and spatial information can be used to reconstruct a volume from a single image. d) A best focus wide field image of the single cell showing partially in focus dendritic structures. e) Three different images recovered from the light field image: 1 \& 2) Single axial planes deconvolved showing individual dendrites seen out of focus in the wide field image. 3) A z-projection through the recovered light field volume image showing the in-focus sections of recovered dendrites. Figure adapted from Quicke (2019) ${ }^{68} \mathrm{CC}$ BY-SA 4.0.

102 transients and compare the extent to which refocused and deconvolved light fields enable lateral

103 and axial transient localization. Finally, we compare temporal signal SNR between LFM and wide

104 field microscopy. 


\section{Methods}

106 This section reproduces methods described in Quicke (2019). ${ }^{68}$ We designed our LFM following

107 the principles set out by Levoy et al. (2006). ${ }^{49}$ We adapted a wide field imaging system by placing

108 a microlens array (MLA) at the microscope image plane, and used a 1:1 relay lens (Nikon $60 \mathrm{~mm}$

109 f/2.8 D AF Micro Nikkor Lens) system to image the MLA back focal plane onto our camera chip

110 (ORCA Flash 4 V2, $2048 \times 2048$ pixels, $6.5 \mu$ m pixel size, Hamamatsu, see Figure 1a.). The lateral

111 resolution is given by the MLA pitch divided by the magnification of the objective. Using our $25 \times$

112 objective (1.0 NA, XLPLN25XSVMP, Olympus) we chose our system to have $5 \mu \mathrm{m}$ lateral pixels,

113 dictating a microlens pitch of $125 \mu \mathrm{m}$.

114 The axial resolution is defined by the number of resolvable diffraction-limited spots behind

115 each microlens. ${ }^{49}$ Assuming a central emission wavelength of $550 \mathrm{~nm}$ for mCitrine, the FRET

116 donor in VSFP-Butterfly $1.2,{ }^{69}$ the spot size in the camera plane is $6.46 \mu \mathrm{m}$ using the Sparrow

117 criterion. With an $125 \mu \mathrm{m}$ pitch MLA, we are able to resolve $N_{u}=19$ distinct spots under each

118 microlens. The depth of field when synthetically refocussing our LFM can therefore be calculated

119 as $7.81 \mu \mathrm{m} .^{49}$

120 To efficiently use the camera sensor, the exit pupil of the objective should map through the

121 MLA to produce circles on the light field plane that are just touching, requiring that the objective

122 image-side f-number (f/12.5) equal the MLA f-number. We chose an f/10 MLA (MLA-S125-f10,

123 RPC Photonics), an off-the-shelf part which came close to matching whilst being a larger aperture.

\subsection{Imaging}

125 This study was carried out in accordance with the recommendations of UK Animals (Scientific

126 Procedures) Act 1986 under Home Office Project and Personal Licenses (project licenses 70/7818 
and 70/9095). Slices were made from 4 mice aged 31, 32, 32 and 175 days transgenically modified to sparsely express VSFP Butterfly $1.2^{69}$ using the method previously described in Song et

129 al. (2017). ${ }^{65,66}$ These transgenic mice express the GEVI in cortical layer 2/3 pyramidal neurons

130 under the intersectional control of TetO and destabilised Cre-recombinase. ${ }^{70-72}$ The destabilised

131 Cre-recombinase was stochastically re-stabilised to induce sparse expression of the voltage indi-

132 cator via two IP injections of a total of $2 \times 10^{-4} \mathrm{mg} \mathrm{kg}^{-1}$ Trimethoprim (TMP, Sigma) over 2

133 consecutive days as described in Song et al. (2017). ${ }^{66}$

134 Slices were prepared at least 2 weeks post TMP injection using a method adapted from Ting et

135 al. (2014) $)^{73}$ (the 'protective recovery' method, www.brainslicemethods.com). $400 \mu \mathrm{m}$ slices were

136 cut with a Camden Microtome 7000 in ice cold 95\% $\mathrm{O}_{2} / 5 \% \mathrm{CO}_{2}$ oxygenated ACSF containing:

137 (in mM) $125 \mathrm{NaCl}, 25 \mathrm{NaHCO}_{3}, 20$ glucose $2.5 \mathrm{KCl}, 1.25 \mathrm{NaH}_{2} \mathrm{PO}_{4}, 2 \mathrm{MgCl}_{2}, 2 \mathrm{CaCl}_{2}$. The

138 slices were then immediately transferred into NMDG-ACSF ${ }^{73}$ containing: (in $\mathrm{mM}$ ) $110 \mathrm{~N}$-methyl-

139 D-Glucamine, $2.5 \mathrm{KCl}, 1.2 \mathrm{NaH}_{2} \mathrm{PO}_{4}, 25 \mathrm{NaHCO}_{3}, 25$ Glucose, $10 \mathrm{MgCl}_{2}, 0.5 \mathrm{CaCl}_{2}$, adjusted

140 to $300-310 \mathrm{mOsm} / \mathrm{Kg}, \mathrm{pH}$ 7.3-7.4 with $\mathrm{HCl}$ and oxygenated with $95 \% \mathrm{O}_{2} / 5 \% \mathrm{CO}_{2}$ at $36{ }^{\circ} \mathrm{C}$ for

14112 minutes before being transferred back into the original sodium-containing ACSF for at least an

142 hour before patching and imaging.

143 Fluorescent cells were patched under oblique infrared illumination $(780 \mathrm{~nm})$ with pipettes of

144 resistances between 3 and $10 \mathrm{MOhms}$ when filled with intracellular solution containing: (in $\mathrm{mM}$ )

145130 K-Gluconate, 7 KCl, 4 ATP - Mg, 0.3 GTP - Na, 10 Phosphocreatine - Na, 10 HEPES. We

146 digitized current clamp signals (Power 1401 digitizer; Cambridge Electronic Design) from a Multi-

147 clamp 700B amplifier (Axon Instruments). At room temperature, we imaged at 100 frames/second

148 for 2.5 seconds whilst injecting current pulses lasting 50 and $100 \mathrm{~ms}$. Each pulse elicited depolar-

149 ization to threshold evoking a single action potential or burst of 2-3 action potentials. We powered 
150 a $490 \mathrm{~nm}$ LED (M490L4, Thorlabs) with a constant current source (Keithley Sourcemeter 1401) to

151 illuminate the sample at $3-11 \mathrm{~mW} / \mathrm{mm}^{2}$. Sets of light field and wide field time series acquisitions

152 were interleaved by removing and replacing the microlens array by hand. We averaged between 4

153 and 16 sweeps per imaging condition. The LED was collimated with an $\mathrm{f}=16 \mathrm{~mm}$ aspheric lens

154 (ACL25416U0-A, Thorlabs) and filtered with a 475/28 nm excitation filter (FITC-EX01-CLIN-25,

155 Semrock). Fluorescence was collected using a $495 \mathrm{~nm}$ long pass dichroic (FF495-Di03, Semrock)

156 along with a 550/88 nm collection filter (FF01-550/88, Semrock) and 496 long pass filter (Sem-

157 rock FF01-496/LP) to attenuate any excitation light transmitted by the dichroic. Imaging data were

158 acquired with Micromanager. ${ }^{74}$ Imaged cells' somata lay between 11 and $40 \mu \mathrm{m}$ below the slice

159 surface, with a median depth of $29 \mu \mathrm{m}$. Data were analysed with custom Python scripts using SciPy

160 packages. $^{75}$

1612.2 Light field reconstruction

162 We reconstructed source volumes using two techniques to compare their performance for single-

163 cell voltage data. We calculated $(\mathrm{x}, \mathrm{y}, \mathrm{z}, \mathrm{t})$ volume time series using synthetic refocussing, ${ }^{49}$ and

164 ISRA $^{53,58}$ using a PSF calculated using the method described in the section below. RL decon-

165 volution $^{50-52}$ was also tested on the data, however little discernible difference in the results was

166 observed.

168 We calculated LFM PSFs differently to previously described, ${ }^{50}$ using the method described in

169 Quicke et al. (2019). ${ }^{67,68}$ Briefly, to calculate the field at the microlens array we considered how

170 a high NA objective lens collects the field from an oscillating electric dipole at position $\mathbf{r}$ near 
171 the microscope focus, $|\mathbf{r}|<<f$, at the origin, calculating the Fourier transform of the field in

172 the objective back focal plane. We assumed that we could model the behaviour of a point source

173 consisting of randomly oriented fluorescent molecules as the incoherent sum of dipoles along 3

174 orthogonal directions. We then used the same method as described in Broxton et al. (2013) ${ }^{50}$ to

175 model transmission through the MLA and to the camera.

176 We calculated the PSF for GEVI imaging deconvolution for $550 \mathrm{~nm}$ emission. We did not

177 oversample the deconvolution as resolving voltage signals generally requires averaging pixels to

178 approximately the native LFM resolution. We therefore generated a single light field kernel for

179 each depth by averaging over kernels sampled for point sources at different lateral positions under

180 the microlens, weighting each point in the average by a 2D Hamming window function of a width

181 equal to our microlens' pitch. We averaged over kernels sampled at 5 times finer than the native

182 microlens resolution. The ISRA was used to deconvolve the data.

\section{$183 \quad 2.2 .2$ Volume reconstructions}

184 Having obtained our downsampled PSF we deconvolved our volume using a similar procedure to

185 previous studies. A key difference is that only a single $2 \mathrm{D}$ convolution was required for each depth

186 in the reconstructed volume for the forwards and backwards projections, respectively, as we did

187 not increase the lateral sampling rate. We applied the deconvolution scheme independently to each

188 frame of the image time sequences, using a cluster to parallelize the data processing. Deconvolu-

189 tion of a single frame took around 30 - 40 minutes for a 21 iteration deconvolution of 21 z-planes

190 on a single CPU. We employed a large cluster to process the individual frames simultaneously,

191 enabling 5000 frames to be processed overnight. We did not use a parallel algorithm within each

192 deconvolution to leverage, e.g., GPU processing, as the computing resources available to us were 
193 better suited to data parallelism. As with previous studies this would greatly increase the rate of

194 individual frames, although it would also likely reduce the number of simultaneous frames that

195 could be deconvolved for typical cluster setups.

196 Synthetic refocusing, based on a ray optics model of light field image formation, is a simpler

197 approach to volume reconstruction that is also much less computationally intensive. Images fo-

198 cused at different $\mathrm{z}$-depths can be constructed by combining individual perspective views using the

199 formula derived in $\mathrm{Ng}$ et al. (2005). ${ }^{76}$ Linear interpolation in this summation results in each pixel

200 being the weighted sum of pixels of the original light field image. This reconstruction is much

201 faster than the iterative deconvolution methods and also does not suffer from noise amplification. ${ }^{77}$

\subsection{Volume time series analysis}

\section{2.3.1 Effect of reconstruction on SNR}

204 To compare the effect of different reconstruction techniques on voltage signal SNR we recon205 structed single planes from volumes at the light field microscope focus. We compared synthetically 206 refocused time series with time series deconvolved using ISRA for different iteration numbers. Re207 gions of interest (ROIs) were manually chosen over the soma and its surround and were identical 208 for the synthetically refocused and deconvolved volumes.

209 As we were collecting fluorescence from the VSFP Butterfly 1.2 FRET donor, fluorescence 210 decreased upon membrane depolarisation. ${ }^{69}$ Therefore the traces shown Figures 2 and 3 are in-

211 verted. To measure SNR we calculated the signal as the $5^{\text {th }}$ percentile value during a stimulus and 212 relaxation period of $200 \mathrm{~ms}$ with the median value of the $100 \mathrm{~ms}$ before the stimulation period 213 subtracted. The noise level was calculated as the standard deviation of a $350 \mathrm{~ms}$ period during no 214 intracellular current stimulus. 


\subsubsection{Depth-time plots}

216 To determine the center of mass of the signal for different cellular ROIs we extracted time courses

217 from each refocused or deconvolved depth, then filtered the resulting depth-time $2 \mathrm{D}$ arrays with a 218 median filter of 11 samples in the time axis $(110 \mathrm{~ms})$ and 3 samples in the $\mathrm{z}$ axis $(15 \mu \mathrm{m})$.

\section{2.3.3 Comparison of light field and wide field SNR}

220 We compared the SNR between trials of the same cell for image sequences taken with wide field 221 and light field imaging systems. We compared the SNR between refocused and wide field images

222 for the same number of repeats using ROIs calculated to be the same for both imaging modalities.

223 For 8/12 cells, an extra aperture was introduced into our light field microscope to compensate

224 for chromatic aberration, reducing the light throughput of the microscope by between $1 / 2$ and 3/4

225 during light field imaging compared to the equivalent wide field trials. To account for this, the SNR

226 for these trials was adjusted by a factor equal to the square root of the ratio of the mean brightness

227 of the first imaging trials from the light field and wide field trials. The microscope was realigned

228 to account for chromatic aberration before the final 4/12 cells, meaning the design light throughput

229 of the microscope was the same between light field and wide field trials. For these trials the raw

230 SNR was included in the analysis.

\section{$231 \quad$ 2.3.4 Signal spread analysis}

232 We compared the lateral and axial signal spread using a method similar to our previous work. ${ }^{65} \mathrm{We}$

233 quantified the neuronal voltage signal strength in each pixel to create 2- or 3-D 'activation maps'

234 by calculating the temporal correlation coefficient of each pixel's time course with a seed time 235 course from the somatic ROI. 
We compared the spatial autocorrelations of these activation maps to quantify the average sig-

237 nal crosstalk between cellular voltage signals. ${ }^{65}$ In our previous work we described how the auto-

238 correlation can be used to quantify the average signal power a cell contributes to a specific pixel's

239 time course and quantified this effect for this preparation with wide field microscopy in 2 dimen-

240 sions. $^{65}$ In this work, we calculated the 3D autocorrelations of the lightfield volumes and $2 \mathrm{D}$

241 autocorrelations of the wide field volumes using Fast Fourier Transform based convolution, setting

242 the central $10 \times 10$ pixels of the autocorrelations to the mean of their perimeter to remove a central

243 noise peak.

\section{Results}

\subsection{Light Field Microscopy Enables Simultaneous Imaging of Axially Separated Dendrites.}

246 We demonstrated LFM's ability to resolve axially separated structures by imaging a cell with a 247 complex 3D dendritic arbour using both wide field and light field microscopy (Fig. 1b) and LFM 248 (Fig. 1c). No single plane wide field image was able to simultaneously bring all the dendrites into 249 a good focus (Fig. 1d), however in different planes from a volume reconstructed by deconvolution 250 different dendritic structures could be clearly distinguished (Fig. 1e1 \& Fig. 1e2). The same 251 cellular features can clearly be seen in a standard deviation projection through the reconstructed 252 LFM stack (Fig. 1e3) and a wide field z stack through the same cell (Fig. 1b, both projections 253 through stacks at $1 \mu \mathrm{m}$ axial increments).

\subsection{Comparison of the Effect of Different Reconstruction Methods on Signal-to-Noise Ratio}

255 Low-sensitivity GEVIs mean SNR is of utmost importance in voltage imaging analysis strategies, 256 and so we first compared the performance of the deconvolution and refocusing reconstruction ap- 

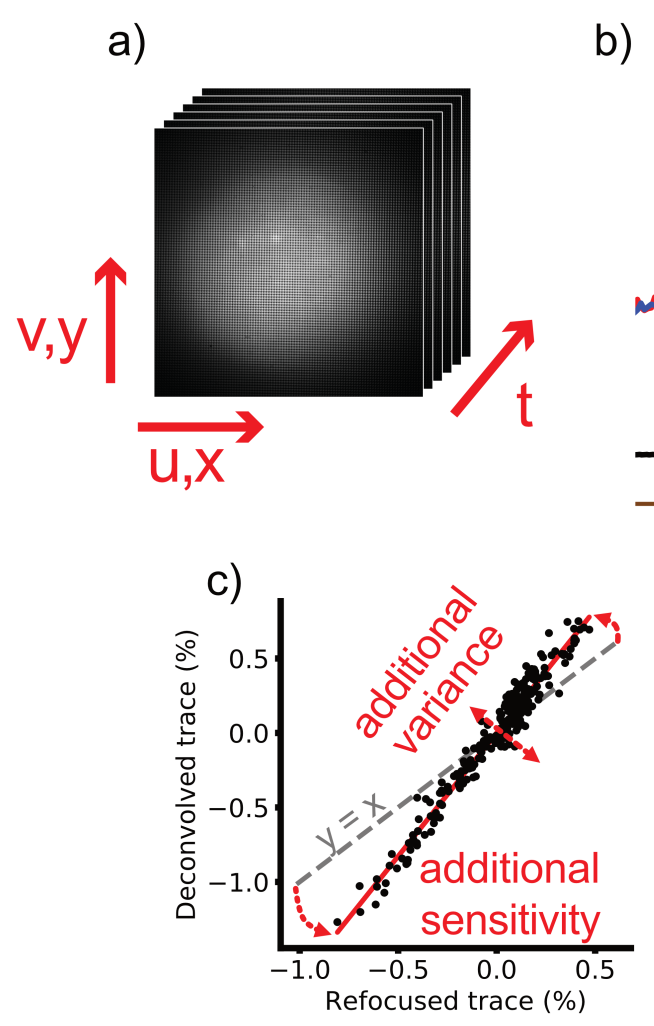

e)

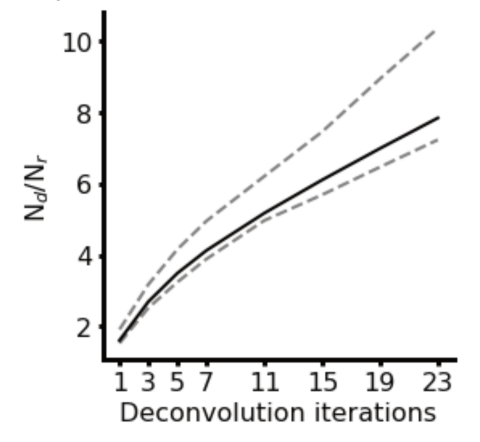

b)
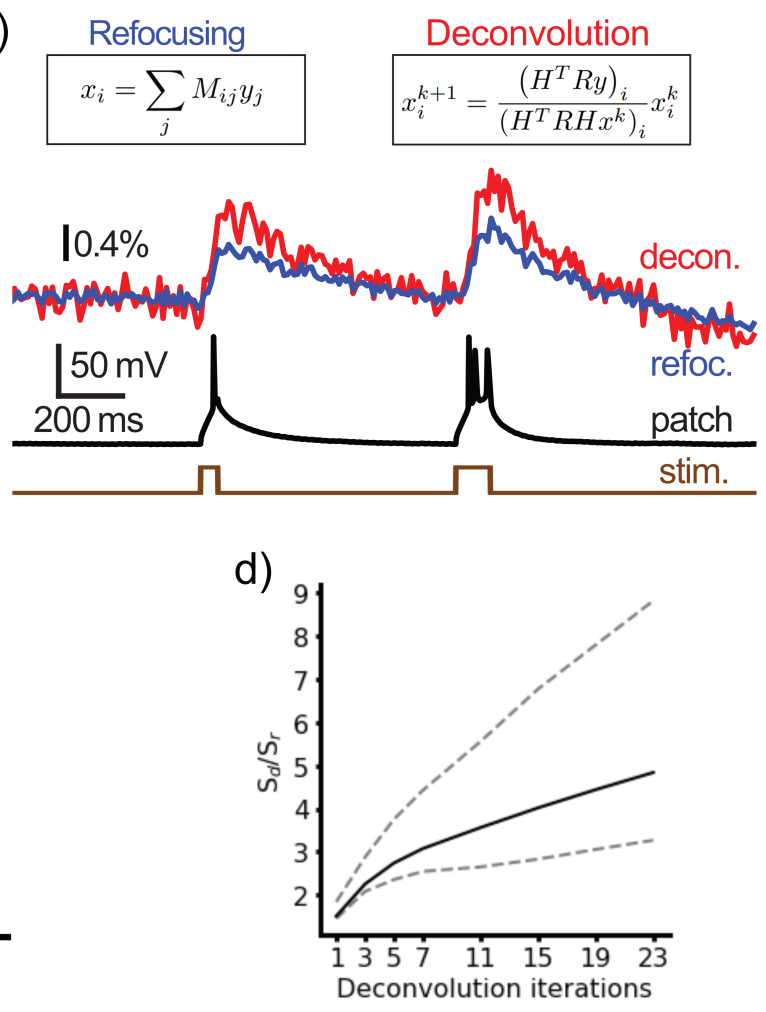

f)

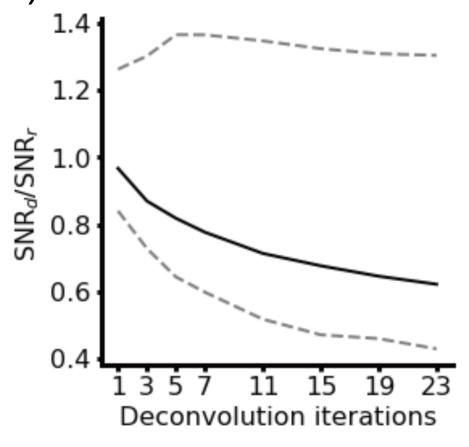

Fig 2 Comparison of different reconstruction methods on SNR. a) Light field time series were collected of functional voltage signals from sparsely expressed GEVIs. b) Time series were extracted from in focus image sequences of the soma via refocusing (left) and ISRA deconvolution (right) and the signal and noise were compared. c) Deconvolved and refocused signals are strongly linearly correlated, as can be seen from plotting the individual trace time points. The additional noise variance due to deconvolution can be identified as the residual from the linear fit. The increased signal level can be seen as the increased fit gradient over unit slope (grey dashed line). Both the noise d) and the signal e) increase monotonically with increasing deconvolution iteration, leading to an overall reduction in SNR with iteration number $\mathrm{f}$ ). At low iteration number the deconvolution and refocusing are very similar. At large iteration number the SNR is decreased relative to refocused; however, increased axial sectioning may still motivate the use of deconvolution methods. Solid lines are median of $n=15$ cells, dashed lines indicate $25^{\text {th }}$ and $75^{\text {th }}$ percentile values. Traces in b) were generated from an average of 8 sweeps. 
257 proaches on this metric. We reconstructed volume time series for 15 cells from LF time series (Fig

258 2a) and extracted optical time courses from ROIs over the individual cell's soma at the native focal

259 plane and compared the SNR between deconvolved and refocused volumes (Fig. 2b). Commonly

260 used LFM iterative reconstruction schemes are prone to noise amplification ${ }^{78}$ which increases with

261 iteration number. It is therefore crucial to understand when to stop the iteration scheme. We used

262 the refocused images as a baseline comparison for the iteration analysis due to the ease of their

263 reconstruction. We found that for all iteration numbers the noise and signal level was increased

264 by deconvolution which increased sensitivity and variance (Figs. 2c - e). The signal significantly

265 increased from $0.3(0.2,0.4) \%$ (all results presented as median (IQR)) in the refocused time series

266 to $1.4(0.9,1.7) \%$ for the 21 iteration deconvolved traces (Wilcoxon signed rank, $n=15, z=0.0$,

$267 p=0.0003)$. The noise significantly increased from $0.05(0.04,0.08) \%$ in the refocused time

268 series to $0.4(0.3,0.5) \%$ for the 21 iteration deconvolved traces (Wilcoxon signed rank, $n=15$,

$269 z=0.0, p=0.0002)$. This resulted in the SNR reducing from approximately the same as the refo-

270 cused $(1.0(0.8,1.3))$ case for a single deconvolution iteration to around half that of the refocused

271 case $(0.6,(0.4,1.3))$. We also processed the light field time series using RL deconvolution and

272 found no substantial differences compared to ISRA.

273 3.3 Light Field Microscopy Resolves 3D Localised and Axially Separated Voltage Signals

274 We then explored a key advantage of sub-cellular resolution light field voltage imaging: 3D imag-

275 ing of neuronal processes. Achieving this requires signals from different planes to be discriminable

276 in volume reconstructions. Axial discriminability depends on intrinsic factors such as depth of

277 field, and also extrinsic factors, such as cellular morphology and signal spread due to tissue scat-

278 tering. To demonstrate the resolution of subcellular voltage transients in 3D, we reconstructed 4D 

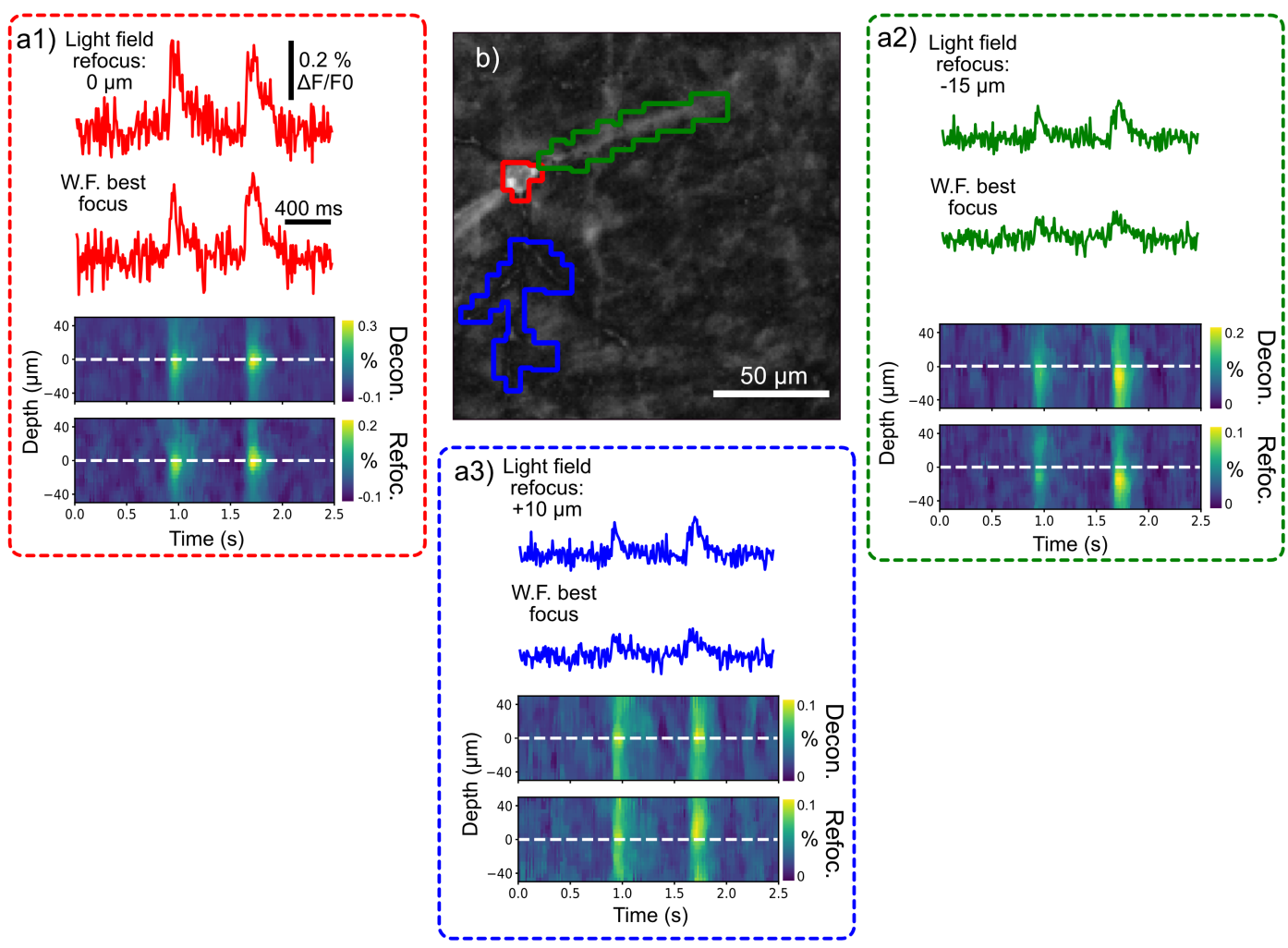

c1)

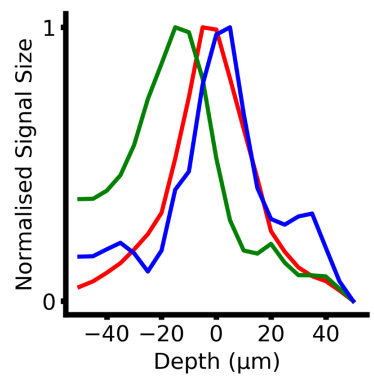

c2)

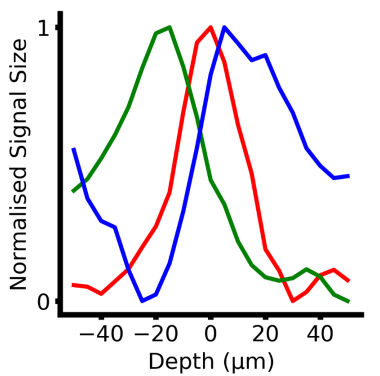

Fig 3 Deconvolution Light field microscopy resolves 3D localised voltage signals. a1-3) Time courses and depth-time plots showing signals from different cellular compartments (shown in (b)) localised at different depths. The somatic signal (a1) is maximal in the wide field and native light field focal planes, whilst the apical dendrite (a2) descends into the slice with its ROI localised 15 um deeper. The signal from a basal dendrite (a3) is superficial to the soma, and its best focal depth is difficult to localize due to the broad axial extent of the refocused signal. The basal and apical dendritic fluorescence transients in the wide field time courses have smaller signals than the light field signals as they are out of plane when focused on the soma. c) The normalised signal size for each ROI across different deconvolved (c1) and refocused (c2) depths. Deconvolution increases the axial localisation of signals. The data are an average of 8 sweeps. 
$279(\mathrm{x}, \mathrm{y}, \mathrm{z}, \mathrm{t})$ volumes from light field image time series and compared the temporal signals from ROIs

280 over different dendritic and somatic structures in multiple axial planes.

281 Figure 3 demonstrates LF imaging's ability to axially localize functional voltage signals from

282 neuronal processes and thereby image functional activity in 3D with SNR unachievable by any

283 equivalent wide field system. Panels 3a 1- 3) show single plane and multi-plane time courses from

284 three different ROIs over cellular compartments from a neuron distributed over multiple axial

285 planes. A somatic ROI (Fig. 3a1) in the native focal plane of both wide field and light field images

286 contains action potential evoked fluorescence transients approximately equal in signal size for both

287 light field and wide field (top). The depth-time plots show the functional signal localised to the

288 native focal plane in the LF functional stacks (bottom). In contrast, an ROI over the apical dendrite

289 (Fig. 3(1) has the largest signal when the LF image is refocused $15 \mu \mathrm{m}$ deeper into the slice, and

290 the signal in the equivalent wide field ROI is much smaller. The depth-time plots for this ROI from

291 both deconvolved and refocused stacks also clearly show the center of mass of the signal located

292 deeper than the native focal plane (Fig. 3(1), bottom). Signals from a basal dendrite (Fig. 3(3)

293 are similarly larger in the LF image refocused $10 \mu \mathrm{m}$ shallower than the native focal plane. The

294 corresponding depth-time plots show a slight shift in the signal center of mass to a shallower depth,

295 especially in the refocused case.

296 Plots of signal size as a function of depth for the refocused and deconvolved cases (Fig. 3c $1 \&$

297 2) show the axial localization as distinctly different planes for each ROI and also demonstrate a key

298 advantage of deconvolved over refocused reconstructions: increased accuracy in axial localization

299 of functional signals. 

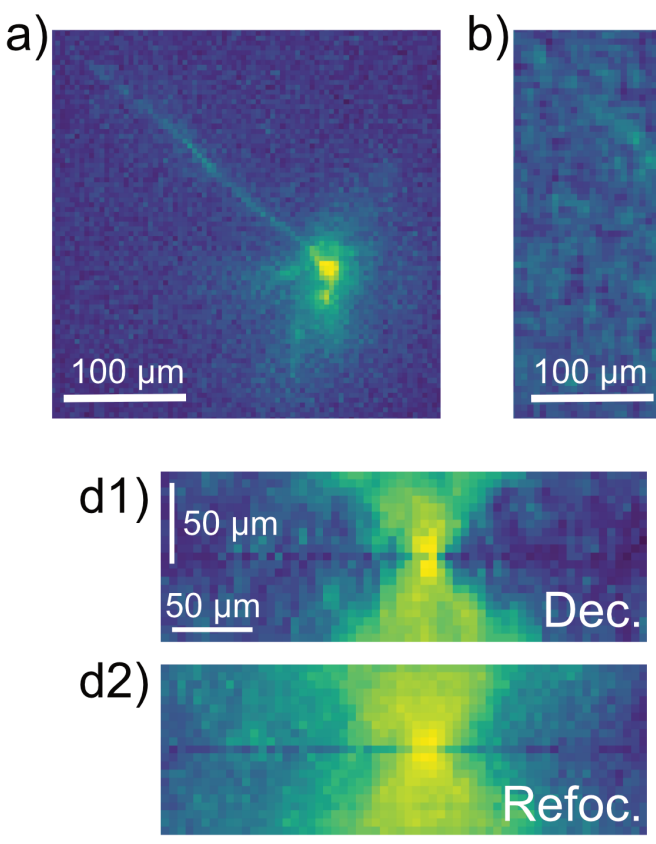

f)

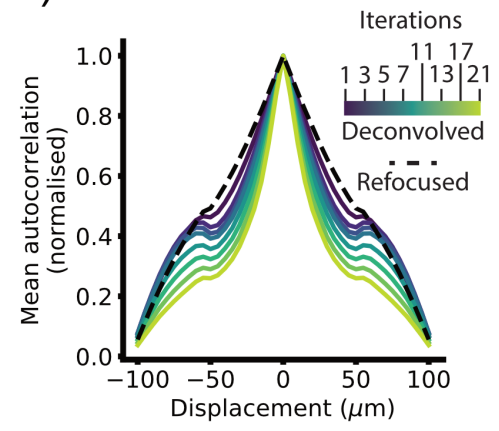

g)

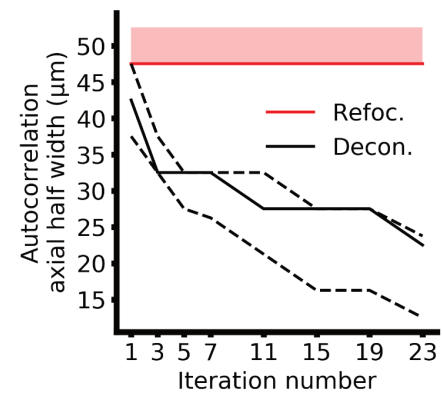

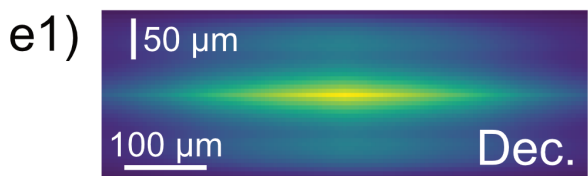

c)

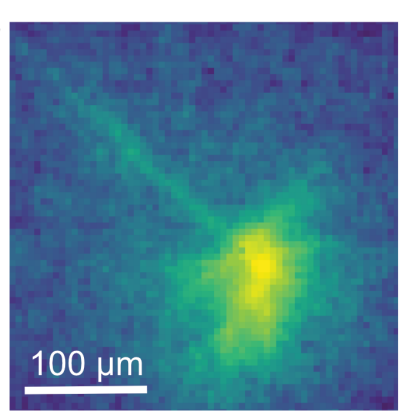

e2)

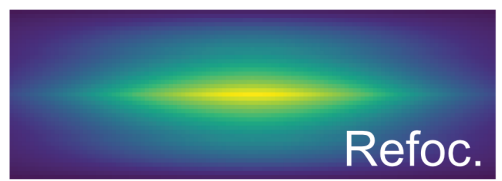

h)

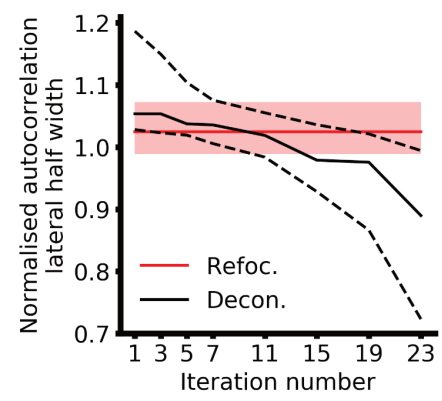

Fig 4 Mapping dendritic signals. a) wide field activation image. b) Deconvolved activation image, sum projection from -35 to +35 um, 5 deconvolution iterations. c) Refocused activation image, sum projection from -35 to +35 um. d) $x-z$ maximum intensity projections through deconvolved (top), and refocused (bottom) activation images showing the different axial sectioning. e) Mean x-z projections through the autocorrelations. f) Normalised maximum autocorrelation for different depths from refocused and deconvolved LFM activation volumes. The secondary peaks arise from the elongated axial PSF, and these can be seen decreasing as the iteration number increases. g) Median autocorrelation axial half widths for $\mathrm{n}=12$ cells with iteration number. Dashed lines represent quartile values. Red line is the refocused median width and shaded area the refocused interquartile range (IQR). h) Median autocorrelation lateral widths normalised to wide field lateral widths for refocused images (red, shaded area IQR) and different deconvolution iterations (black lines, dashed lines IQR). 


\subsection{Deconvolution Increases Axial Localisation of Functional Voltage Signals}

301 The transients from refocused volumes exhibit a larger axial PSF width compared to the decon-

302 volved traces (Fig. 3c). Hence these signals are smeared out, reducing distinguishability of signal

303 contributions from different planes. To quantify this effect we generated volumes showing the

304 distribution of functional signal. We generated a time course from an in focus somatic ROI and

305 calculated the temporal correlation coefficient of every pixel in the volume for refocused and de-

306 convolved volume time series. Pixels with high correlation coefficients are interpreted as having a

307 large response to the intracellular current injection, and so a volume map of these reveals morphol-

308 ogy of structures through which the functional signal propagates. Activation maps from wide field

309 imaging trials show blurring around the soma from out of focus basal dendrites (Fig. 4a). Com-

310 paratively, z-projections from a $70 \mu \mathrm{m}$ region around the soma generated from the deconvolved

311 activation volume (Fig. 4b) reveals the structures that cause this blur. A projection through $70 \mu \mathrm{m}$

312 around the focus from the refocused case shows significantly more blurring due to the poor axial

313 sectioning of this technique (Fig. 4c). We used the 3D autocorrelation of these activation maps to

314 quantify the spread of the signal in 3D (see section 2.3.4). We quantified how the peak autocorre-

315 lation from each cell, and therefore functional signal contribution, decayed axially. Axial smearing

316 can be seen in reconstructions from both deconvolution and refocusing (Fig. 4d), although the ef-

317 fect is much more severe in refocused traces. The smearing appears in the axial autocorrelation as

318 both broadening of the central peak and increased side lobes (Fig. 4e). The central peak width and

319 side lobes decrease with increased deconvolution iteration number (Fig. 4f \& g), thus increasing

320 the axial sectioning.

321 The autocorrelation widths decreased significantly from 1 to 21 iterations (median dropped 
322 from $42.5(37.5,47.5) \mu \mathrm{m}$ to $22.5(12.5,23.75) \mu \mathrm{m}, z=0, p=0.002)$, and for both cases the

323 axial spread was significantly lower than refocused (median of $47.5(47.5,52.5) \mu \mathrm{m}, p=0.001$,

$324 p=0.002$ for 1 and 21 iterations respectively). Significance tests were performed with a Friedman

$325 \chi^{2}$ with post hoc Bonferroni-corrected Wilcoxon signed-rank tests (significant at $p<0.017$ ). $\mathbf{n}=$

32612 cells from 12 slices from 4 mice. Friedman $\chi^{2}=24, p=6 \times 10^{-6}$.

327 Finally we compared how deconvolution and refocusing affected lateral signal localization compared to the equivalent wide field time series (Fig. 4h). We measured the width of radially averaged autocorrelations normalised to matched wide field trials for the refocused and deconvolved cases. We found that the lateral signal spread significantly decreased from 1 to 21 iterations (median dropped from $1.05(1.03,1.18)$ times larger than wide field trials to $0.89(0.73,0.99)$ times

332 larger, $Z=0, p=0.002$ ), from significantly larger than the WF and refocused at 1 iteration

$333(z=1, p=0.003$ and $z=0, p=0.002$ respectively), to significantly smaller than the refocused

334 at 21 iterations $(z=0.0, p=0.003)$. The refocused widths did not differ significantly from the 335 matched wide field trials (median $1.02(0.99,1.07)$ times larger, $z=17, p=0.08)$. Significance 336 tests were performed with a Friedman $\chi^{2}$ with post hoc Bonferroni-corrected Wilcoxon signed337 rank tests on the raw widths (significant at $p<0.0083$ ). $n=12$ cells from 12 slices from 4 mice. 338 Friedman $\chi^{2}=26, p=9 \times 10^{-6}$.

339 In total our analyses reveal that deconvolution improves both axial and lateral signal local340 ization, but decreases temporal signal SNR compared to synthetic refocussing, with both effects 341 intensifying with increasing iteration number. 


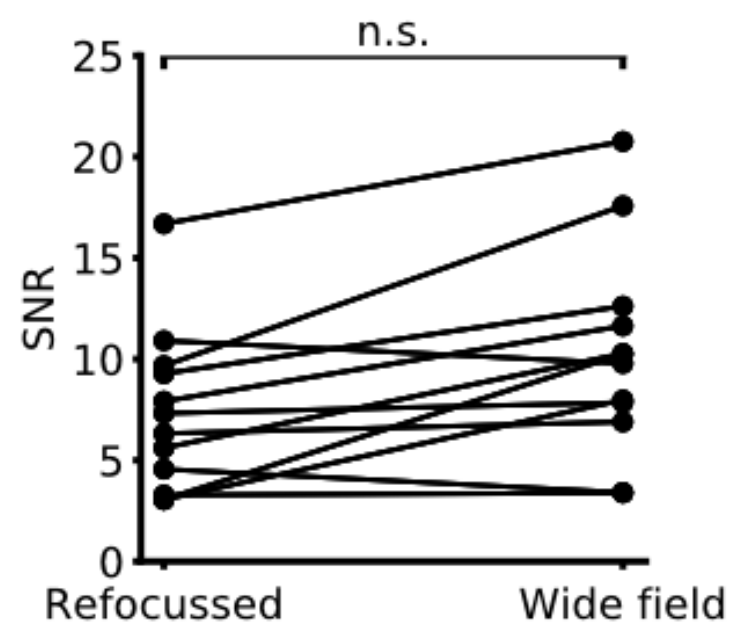

Fig 5 Comparison of Light field and Wide Field SNR. Points correspond to mean SNR between paired light field and wide field trials. Light field microscopy SNR does not differ significantly from wide field. For 8/12 trials we included a correction factor due to a misalignment in the LFM as discussed in section 2.3.3.

343 We measured the SNR for paired wide field and refocused light field imaging trials in the same

344 cells. For 8/12 trials we included a correction factor due to a misalignment in the LFM as discussed

345 in section 2.3.3. The SNR did not change significantly between the light field and wide field cases

346 (Wilcoxon signed rank test, $\mathrm{n}=12$ cells from 12 slices and 4 mice, $z=32, p=0.6$ ), with a median

347 light field SNR of $8.4(5.2,11.4)$ and a median wide field SNR of $10.0(7.6,11.9)$.

\section{Discussion}

349 We have shown that LFM enables 3D sub-cellular GEVI imaging of somatic and dendritic struc-

350 tures. We demonstrated that LFM enables simultaneous imaging of axially separated dendrites,

351 overcoming a key limitation of wide field imaging. We further showed that functional voltage

352 signals from dendrites could be axially resolved at different depths. This finding is key to demon-

353 strating LFM's utility for studies of dendritic integration or synaptic mapping.

354 We compared how synthetic refocussing and deconvolution-based reconstruction techniques 
perform with respect to spatial signal localization and temporal SNR. Synthetic refocussing is

356

358 computationally simple and can be used to process light fields online, during an experiment, or post hoc. Refocusing features better temporal signal SNR but poorer lateral and axial confinement compared to deconvolution. Deconvolution has two major disadvantages: computational cost and noise amplification. As the light field microscope PSF is not shift invariant it is described by a 5 dimensional matrix, complicating reconstruction. The periodicity it displays under lateral shifts by integer multiples of the microlens pitch, however, enable deconvolution to be performed efficiently by using FFT-based convolutions. Despite this, even small increases in lateral sampling in the deconvolved volume increase the computational cost of reconstruction drastically. Reconstructing $n_{z} z$-planes in a volume with a lateral increase in sampling over the native LFM sampling of $m$ requires $2 \times n_{z} \times m^{2} 2 \mathrm{D}$ convolutions per iteration, precluding online image processing. Secondly, both Richardson-Lucy and ISRA tend to amplify noise in their outputs due to their lack of regularization. ${ }^{78}$ This noise may be acceptable when imaging high-SNR calcium signals, however it can dominate small, dim voltage signals. Incorporating regularization into the deconvolution approaches to suppress noise overfitting could also ameliorate deconvolution's effects on temporal SNR.

In this study we imaged VSFP-Butterfly 1.2, an older generation probe. GEVI technology has advanced dramatically recently, greatly increasing their sensitivity, and with these new sensors noise amplification due to deconvolution in the light field volume reconstruction may become less significant. Although VSFP-Butterfly 1.2 exhibits lower sensitivity than several recently reported probes, ${ }^{20,79-83}$ we were able to express it sparsely and strongly to enable single-cell GEVI imag-

76 ing without somatic restriction, which would preclude study of sub-cellular signals. ${ }^{65,66}$ The slow 377 kinetics of the probe used in this study also enabled resolution of action potentials at 100 frames/s 

without severe aliasing. Although we could resolve single-sweep signals, signal averaging was required to resolve smaller dendritic signals with adequate SNR. With a more recent GEVI, dendritic processes could likely be resolved in single sweeps.

Newer voltage sensors can not immediately be combined with LFM, however, as they require much faster sampling rates, typically between 500 - $1000 \mathrm{~Hz}$. Megapixel cameras with $1 \mathrm{kHz}$ fullframe readout rates are therefore needed to fully exploit these newer voltage indicators. Current sCMOS cameras such as the one used in this study can achieve these imaging rates by reducing the FOV to a small central strip of the image sensor. This, however, is particularly detrimental to LFM compared to wide field imaging as the LFM PSF spreads information about each point widely across the image sensor for objects away from the focal plane. If only a small strip of the sensor is imaged SNR will be greatly degraded as light is lost outside of this reduced FOV. We anticipate that this issue will be steadily ameliorated as faster sCMOS sensor technology is developed.

A second issue arises with newer, faster GEVIs due to their requirement for much faster frame rates. Deconvolving individual frames with these sensors would require a drastic increase in computational resources and is likely untenable. Approaches have been developed for calcium imaging light field time series which do not involve deconvolution of every frame. ${ }^{61,84}$ In their current form, however, these are unsuitable for reconstruction of subcellular light field voltage imaging time series as they leverage the temporal and spatial characteristics of neuronal calcium imaging as reconstruction priors. These priors, such as somatic signal localisation or sparse temporal activity, are not as applicable to subcellular voltage imaging signals, which are smaller, less temporally sparse and arise from more morphologically intricate structures than neuronal somata.

Finally, in this study we compared the SNR between refocused LFM volumes and matched wide field traces and found they did not differ significantly. This is expected, as apart from light 
401 losses at the MLA, which are $<15 \%$ according to the manufacturer, there are no significant losses of SNR to shot noise between wide field and light field microscopy. Together these results have the potential to motivate further work and widespread application of light field microscopy to voltage

404 imaging owing to light fields high photon budget and ability to resolve neurons in three spatial 405 dimensions.

\section{Disclosures}

407 The authors declare that the research was conducted in the absence of any commercial or financial 408 relationships that could be construed as a potential conflict of interest.

\section{Acknowledgements}

410 We thank Yu Liu for her technical assistance and support for research in the Foust and Schultz 411 labs. The authors would also like to thank the Imperial College Research Computing Service.

412 This work was supported by the following grants: Engineering and Physical Sciences Research 413 Council (EP/L016737/1); Wellcome Trust Seed Award (201964/Z/16/Z); The Royal Academy of 414 Engineering under the RAEng Research Fellowships scheme (RF1415/14/26); Biotechnology and 415 Biology Research Council (BB/R009007/1 and BB/R022437/1); The Royal Society (TA/R1/170047);

416 The BRAIN initiative (US National Institutes of Health, U01MH109091, U01NS099573).

\section{Author Contributions}

418 PQ, CLH, SRS and AJF conceived and designed the experiments. CLH and AJF designed the 419 light field optics. PQ and CLH performed experiments. PQ, CLH, AJF and SRS designed the 420 analysis. PQ, HVJ, PS, and PLD developed the deconvolution approach. CS and TK developed 421 and prepared animals with strong GEVI expression limited to a sparse neuronal subpopulation. 

authors contributed to manuscript revision and approved the final manuscript.

\section{Data, Materials, and Code Availability}

The datasets and code generated for this study are available on request to the corresponding author.

\section{References}

1 A. Foust, M. Popovic, D. Zecevic, et al., "Action Potentials Initiate in the Axon Initial Segment and Propagate through Axon Collaterals Reliably in Cerebellar Purkinje Neurons," The Journal of Neuroscience 30(20), 6891-6902 (2010).

2 A. J. Foust, Y. Yu, M. Popovic, et al., "Somatic membrane potential and kv1 channels control spike repolarization in cortical axon collaterals and presynaptic boutons," Journal of Neuroscience 31(43), 15490-15498 (2011).

3 M. A. Popovic, X. Gao, N. T. Carnevale, et al., "Cortical dendritic spine heads are not electrically isolated by the spine neck from membrane potential signals in parent dendrites," Cerebral Cortex 24, 385-395 (2014).

4 K. Holthoff, D. Zecevic, and A. Konnerth, "Rapid time course of action potentials in spines and remote dendrites of mouse visual cortex neurons," Journal of Physiology 588, 1085-1096 tion potentials and synaptic input.," The Journal of neuroscience : the official journal of the Society for Neuroscience 29(21), 6897-6903 (2009). 

(2011).

7 M. Djurisic, S. Antic, W. R. Chen, et al., "Voltage imaging from dendrites of mitral cells: Epsp attenuation and spike trigger zones," Journal of Neuroscience 24(30), 6703-6714 (2004).

8 C. J. Roome and B. Kuhn, "Simultaneous dendritic voltage and calcium imaging and somatic recording from purkinje neurons in awake mice," Nature communications 9(1), 1-14 (2018).

9 M. Canepari, S. Willadt, D. Zecevic, et al., "Imaging inhibitory synaptic potentials using voltage sensitive dyes," Biophysical journal 98(9), 2032-2040 (2010).

10 S. D. Antic, R. M. Empson, and T. Knöpfel, "Voltage imaging to understand connections and functions of neuronal circuits," Journal of neurophysiology 116(1), 135-152 (2016).

11 T. Kwon, M. Sakamoto, D. S. Peterka, et al., "Attenuation of synaptic potentials in dendritic spines," Cell reports 20(5), 1100-1110 (2017).

12 S. Chamberland, H. H. Yang, M. M. Pan, et al., "Fast two-photon imaging of subcellular voltage dynamics in neuronal tissue with genetically encoded indicators," Elife 6, e25690

13 S. D. Antic, "Action potentials in basal and oblique dendrites of rat neocortical pyramidal (2017). neurons," The Journal of physiology 550(1), 35-50 (2003).

14 S. Antic, G. Major, and D. Zecevic, "Fast optical recordings of membrane potential changes from dendrites of pyramidal neurons," Journal of neurophysiology 82(3), 1615-1621 (1999). 

of Sciences 108(49), 19504-19509 (2011).

16 S. Quirin, N. Vladimirov, C.-T. Yang, et al., "Calcium imaging of neural circuits with extended depth-of-field light-sheet microscopy,” Optics letters 41(5), 855-858 (2016).

17 R. Tomer, M. Lovett-Barron, I. Kauvar, et al., "Sped light sheet microscopy: fast mapping of biological system structure and function," Cell 163(7), 1796-1806 (2015). responses of cortical neurons genetically encoded with a fluorescent voltage sensor in rat," Frontiers in neural circuits 6, 15 (2012).

19 W. Akemann, M. Sasaki, H. Mutoh, et al., "Two-photon voltage imaging using a genetically encoded voltage indicator," Scientific reports 3, 2231 (2013).

20 V. Villette, M. Chavarha, I. K. Dimov, et al., "Ultrafast two-photon imaging of a high-gain voltage indicator in awake behaving mice," Cell 179(7), 1590-1608 (2019). 23(9), 655-657 (1998).

22 K. H. Kim, C. Buehler, K. Bahlmann, et al., "Multifocal multiphoton microscopy based on multianode photomultiplier tubes," Optics express 15(18), 11658-11678 (2007).

23 T. Nielsen, M. Fricke, D. Hellweg, et al., "High efficiency beam splitter for multifocal multiphoton microscopy," Journal of microscopy 201(3), 368-376 (2001). (2012). 
25 P. Quicke, S. Reynolds, M. Neil, et al., "High speed functional imaging with source localized multifocal two-photon microscopy," Biomedical Optics Express 9, 3678 (2018).

26 J. Wu, Y. Liang, S. Chen, et al., "Kilohertz two-photon fluorescence microscopy imaging of neural activity in vivo," Nature Methods 17(3), 287-290 (2020).

27 T. Zhang, O. Hernandez, R. Chrapkiewicz, et al., "Kilohertz two-photon brain imaging in awake mice," Nature methods 16(11), 1119-1122 (2019).

28 R. Prevedel, A. J. Verhoef, A. J. Pernía-Andrade, et al., "Fast volumetric calcium imaging across multiple cortical layers using sculpted light.," Nature methods 13, 1021-1028 (2016).

29 S. Weisenburger, F. Tejera, J. Demas, et al., "Volumetric ca2+ imaging in the mouse brain using hybrid multiplexed sculpted light microscopy," Cell 177(4), 1050-1066 (2019).

30 M. Žurauskas, S. Waddell, M. Frade-Rodriguez, et al., "Rapid adaptive remote focusing microscope for sensing of volumetric neural activity," Biomedical Optics Express 8, 4369 (2017).

31 A. Kazemipour, O. Novak, D. Flickinger, et al., "Kilohertz frame-rate two-photon tomography," Nature methods 16(8), 778 (2019).

32 H. Dana, A. Marom, S. Paluch, et al., "Hybrid multiphoton volumetric functional imaging of large-scale bioengineered neuronal networks," Nature communications 5, 3997 (2014).

33 M. B. Ahrens, M. B. Orger, D. N. Robson, et al., "Whole-brain functional imaging at cellular resolution using light-sheet microscopy," Nat Methods 10(5), 413-420 (2013).

34 J. Huisken, J. Swoger, F. Del Bene, et al., "Optical sectioning deep inside live embryos by selective plane illumination microscopy," Science 305(5686), 1007-1009 (2004). 
35 P. J. Keller and M. B. Ahrens, "Visualizing whole-brain activity and development at the single-cell level using light-sheet microscopy," Neuron 85(3), 462-483 (2015).

36 M. B. Bouchard, V. Voleti, C. S. Mendes, et al., "Swept confocally-aligned planar excitation (SCAPE) microscopy for high-speed volumetric imaging of behaving organisms," Nature Photonics 9, 113-119 (2015).

37 D. Oron, E. Tal, and Y. Silberberg, "Scanningless depth-resolved microscopy," Optics express 13(5), 1468-1476 (2005).

38 G. Zhu, J. Van Howe, M. Durst, et al., "Simultaneous spatial and temporal focusing of femtosecond pulses," Optics express 13(6), 2153-2159 (2005).

39 T. Schrödel, R. Prevedel, K. Aumayr, et al., "Brain-wide 3d imaging of neuronal activity in caenorhabditis elegans with sculpted light," Nature methods 10(10), 1013 (2013).

40 C. J. Rowlands, D. Park, O. T. Bruns, et al., "Wide-field three-photon excitation in biological samples," Light: Science \& Applications 6, e16255-e16255 (2017).

41 O. Therrien, B. Aubé, S. Pagès, et al., "Wide-field multiphoton imaging of cellular dynamics in thick tissue by temporal focusing and patterned illumination," Biomedical optics express 2(3), 696-704 (2011).

42 A. J. Foust, V. Zampini, D. Tanese, et al., "Computer-generated holography enhances voltage dye fluorescence discrimination in adjacent neuronal structures," Neurophotonics 2(2), 021007 (2015).

43 V. Nikolenko, B. O. Watson, R. Araya, et al., "Slm microscopy: scanless two-photon imaging and photostimulation using spatial light modulators," Frontiers in neural circuits 2, 5 (2008). 
44 S. Bovetti, C. Moretti, S. Zucca, et al., "Simultaneous high-speed imaging and optogenetic inhibition in the intact mouse brain," Scientific reports 7, 40041 (2017).

45 D. Tanese, J.-Y. Weng, V. Zampini, et al., "Imaging membrane potential changes from dendritic spines using computer-generated holography," Neurophotonics 4(3), 031211 (2017).

46 E. J. Botcherby, R. Juškaitis, and T. Wilson, "Scanning two photon fluorescence microscopy with extended depth of field," Optics Communications 268, 253-260 (2006).

47 R. Lu, W. Sun, Y. Liang, et al., "Video-rate volumetric functional imaging of the brain at synaptic resolution," Nature Neuroscience 20, 620-628 (2017).

48 A. Song, A. S. Charles, S. A. Koay, et al., "Volumetric two-photon imaging of neurons using stereoscopy (vtwins)," Nature methods 14(4), 420-426 (2017).

49 M. Levoy, R. Ng, A. Adams, et al., "Light field microscopy," in ACM SIGGRAPH 2006 Papers on - SIGGRAPH '06, 25(3), 924, ACM Press, (New York, New York, USA) (2006).

50 M. Broxton, L. Grosenick, S. Yang, et al., "Wave optics theory and 3-D deconvolution for the light field microscope," Optics Express 21(21), 25418 (2013).

51 W. H. Richardson, "Bayesian-Based Iterative Method of Image Restoration*," Journal of the Optical Society of America 62, 55 (1972).

52 L. B. Lucy, "An iterative technique for the rectification of observed distributions," The Astronomical Journal 79, 745 (1974).

53 M. E. Daube-Witherspoon and G. Muehllehner, “An Iterative Image Space Reconstruction Algorithm Suitable for Volume ECT," IEEE Transactions on Medical Imaging 5, 61-66 (1986). 
54854 H. Li, C. Guo, D. Kim-Holzapfel, et al., "Fast, volumetric live-cell imaging using high-

549 resolution light-field microscopy," Biomedical Optics Express 10, 29 (2019).

55055 C. Guo, W. Liu, X. Hua, et al., "Fourier light-field microscopy," Opt. Express 27, 25573$55125594(2019)$.

55256 X. Lin, J. Wu, G. Zheng, et al., "Camera array based light field microscopy," Biomedical $553 \quad$ optics express 6(9), 3179-3189 (2015).

55457 N. Cohen, S. Yang, A. Andalman, et al., "Enhancing the performance of the light field mi555 croscope using wavefront coding," Optics express 22(20), 24817-24839 (2014).

58 R. Prevedel, Y.-G. Yoon, M. Hoffmann, et al., "Simultaneous whole-animal 3D imaging of neuronal activity using light-field microscopy.," Nature methods 11(7), 727-30 (2014).

59 N. Wagner, N. Norlin, J. Gierten, et al., "Instantaneous isotropic volumetric imaging of fast biological processes," Nature methods 16(6), 497-500 (2019).

60 L. M. Grosenick, M. Broxton, C. K. Kim, et al., "Identification Of Cellular-Activity Dynamics Across Large Tissue Volumes In The Mammalian Brain,” bioRxiv, 132688 (2017).

61 T. Nöbauer, O. Skocek, A. J. Pernía-Andrade, et al., "Video rate volumetric Ca2+ imaging across cortex using seeded iterative demixing (SID) microscopy," Nature Methods 14, 811818 (2017).

62 O. Skocek, T. Nöbauer, L. Weilguny, et al., "High-speed volumetric imaging of neuronal activity in freely moving rodents," Nature Methods 15, 429-432 (2018). 
64 L. Cong, Z. Wang, Y. Chai, et al., "Rapid whole brain imaging of neural activity in freely behaving larval zebrafish (Danio rerio)," eLife 6 (2017).

65 P. Quicke, C. Song, E. J. McKimm, et al., "Single-neuron level one-photon voltage imaging with sparsely targeted genetically encoded voltage indicators.," Frontiers in Cellular Neuroscience 13, 39 (2019).

66 C. Song, Q. Do, S. Antic, et al., “Transgenic Strategies for Sparse but Strong Expression of Genetically Encoded Voltage and Calcium Indicators," International Journal of Molecular Sciences 18, 1461 (2017).

67 P. Quicke, C. L. Howe, P. Song, et al., "Calculation of high numerical aperture lightfield microscope point spread functions," in Imaging and Applied Optics 2019 (COSI, IS, MATH, pcAOP), CW4A.2, OSA, (Washington, D.C.) (2019).

68 P. Quicke, Improved methods for functional neuronal imaging with genetically encoded voltage indicators. PhD thesis, Imperial College London (2019).

69 W. Akemann, H. Mutoh, A. Perron, et al., "Imaging neural circuit dynamics with a voltagesensitive fluorescent protein.," Journal of neurophysiology 108, 2323-37 (2012).

70 M. Mayford, M. E. Bach, Y. Y. Huang, et al., "Control of memory formation through regulated expression of a CaMKII transgene.," Science (New York, N.Y.) 274(5293), 1678-1683 (1996).

71 J. A. Harris, K. E. Hirokawa, S. A. Sorensen, et al., "Anatomical characterization of Cre driver mice for neural circuit mapping and manipulation," Frontiers in Neural Circuits 8, 76 (2014).

72 L. Madisen, A. R. Garner, D. Shimaoka, et al., “Transgenic Mice for Intersectional Targeting 
of Neural Sensors and Effectors with High Specificity and Performance," Neuron 85, 942958 (2015).

73 J. T. Ting, T. L. Daigle, Q. Chen, et al., "Acute brain slice methods for adult and aging animals: application of targeted patch clamp analysis and optogenetics.," Methods in molecular biology (Clifton, N.J.) 1183, 221-42 (2014).

74 A. D. Edelstein, M. A. Tsuchida, N. Amodaj, et al., "Advanced methods of microscope control using $\mu$ Manager software.," Journal of biological methods 1(2), 10 (2014).

75 P. Virtanen, R. Gommers, T. E. Oliphant, et al., "SciPy 1.0: Fundamental Algorithms for Scientific Computing in Python," Nature Methods 17, 261-272 (2020).

76 R. Ng, M. Levoy, M. Brédif, et al., "Light Field Photography with a Hand-Held Plenoptic Camera - Stanford Tech Report CTSR 2005-02,” tech. rep. (2005).

77 E. Thiébaut, "Introduction to image reconstruction and inverse problems," in NATO Science Series II., Mathematics, Physics and Chemistry, R. Foy and F.-C. Foy, Eds., 397, Springer (2005).

78 N. Dey, L. Blanc-feraud, C. Zimmer, et al., "Richardson-Lucy Algorithm with Total Vari606 ation Regularization for 3D Confocal Microscope Deconvolution," Microscopy Research \&

79 A. S. Abdelfattah, T. Kawashima, A. Singh, et al., "Bright and photostable chemigenetic 609 indicators for extended in vivo voltage imaging," Science 365(6454), 699-704 (2019). dependent changes in hippocampal dynamics," Nature 569(7756), 413-417 (2019). 
61281 K. D. Piatkevich, E. E. Jung, C. Straub, et al., "A robotic multidimensional directed evolution

613 approach applied to fluorescent voltage reporters," Nature Chemical Biology 14, 352-360

614 (2018).

61582 B. Yi, B. E. Kang, S. Lee, et al., "A dimeric fluorescent protein yields a bright, red-shifted

616 GEVI capable of population signals in brain slice," Scientific Reports 8(1), 15199 (2018).

61783 M. Kannan, G. Vasan, C. Huang, et al., "Fast, in vivo voltage imaging using a red fluorescent 618 indicator," Nature methods 15(12), 1108-1116 (2018).

61984 N. C. Pégard, H.-Y. Liu, N. Antipa, et al., "Compressive light-field microscopy for 3d neural 620 activity recording," Optica 3(5), 517-524 (2016). 